\title{
Investigation of Methylmercury and Total Mercury Contamination in Mollusk Samples Collected from Coastal Sites along the Chinese Bohai Sea
}

\author{
Li-na Liang, Jian-bo Shi, Bin He, Gui-Bin Jiang, * and Chun-Gang Yuan \\ Key Laboratory of Environmental Chemistry and Ecotoxicology, Research Center for \\ Eco-Environmental Sciences, Chinese Academy of Sciences, P.O. Box 2871, 100085 Beijing, China
}

\begin{abstract}
This paper presents the investigation results of methylmercury and total mercury in gastropod and bivalve species collected from eight coastal sites along the Chinese Bohai Sea. The total mercury was directly determined by atomic fluorescence spectrometry (AFS), while the methylmercury was measured by a laboratory established high performance liquid chromatography-atomic fluorescence spectrometry system (HPLC-AFS). Certified reference material DORM-2 (Dogfish muscle) was used to validate the two methods and the obtained results proved to be in good agreement with the certified values. It was demonstrated that the mercury contamination was commonly existed in all selected mollusks, with methylmercury and total mercury concentration in the range of 4.8-168.4 and 6.7$453.0 \mathrm{ng} \mathrm{Hg} \mathrm{g}{ }^{-1}$, respectively. Mollusks from HuLuDao were the most mercury contaminated, and those from PengLai took the second place. The species-dependent bioaccumulation capacity was observed in this study. Gastropods showed more capacity to bioaccumulate mercury than bivalves, and mercury contents in two kinds of gastropods (Rapana venosa and Neverita didyma) presented some uplifted trends with the dimensions increasing of the gastropods. Rapana venosa was found to be a potential biomarker to monitor mercury pollution in oceans. Evaluations were also made concerning about the ratio of methylmercury to total mercury.
\end{abstract}

KEYWORDS: Methylmercury; total mercury; HPLC-AFS; mollusks

\section{INTRODUCTION}

Mercury is an important but toxic element. Both the direct drainage of industrial wastewater and rainwater runoff leads to mercury contamination in oceans. Mercury is first absorbed by phytoplankton and then by various consumers within the oceanic ecosystem. Mollusks lie in the second trophic level in this ecosystem and accumulate less methylmercury than predatory fish. However, mollusks are popular seafood, and long-term consumption of them may result in methylmercury accumulation in the human body.

The toxicity of mercury is well known since the notorious poisoning accident at Minamata Bay in southern Japan during the 1950s and 1960s. This tragic event was due to the consumption of methylmercury-contaminated fish, and 48 persons died. Acute exposure to methylmercury is lethal, and chronic low-dose consumption of methylmercury-contaminated fish can cause severe adverse effects to organs, the central nervous system, and the immune system. Consumption of mercury-contaminated fish is particularly hazardous for pregnant women because of its heredity effect on the unborn fetus. Its virulence effect cannot be eradicated for many years.

*Corresponding author. Fax: 8610-62849179. E-mail: gbjiang@ mail.rcees.ac.cn.
Because methylmercury is the most toxic form of mercury, it is now more appropriate to determine not only the total mercury content of seafood but also the methylmercury content. Gas chromatography (GC) separation coupled with electron capture detection (ECD) proposed by Westöö (1) was the commonly used method for the determination of methylmercury. More recently, high performance liquid chromatography (HPLC) hyphenated with atomic fluorescence detector (AFS) has become popular $(2-4)$.

In this present investigation, the contents of methylmercury and total mercury of 88 mollusks samples collected from eight coastal sites were analyzed. The methylmercury was determined with our previously proposed HPLC-AFS method (4), but the sample preparation was slightly modified in order to shorten the preparation time. The AFS method was adopted for the total mercury determination. It was found that the ratio of methylmercury to total mercury varied slightly between gastropods and bivalves, and Rapana venosa was found to be a potential biomarker to monitor the mercury pollution in ocean.

\section{MATERIALS AND METHODS}

Instrumentation. The HPLC (LC-10AT vp, Shimadzu, Japan) and AFS (AF-610A, Beijing Raileigh Analytical Instrument Co., China) hyphenation system was as described previously (4). The AFS peristaltic 


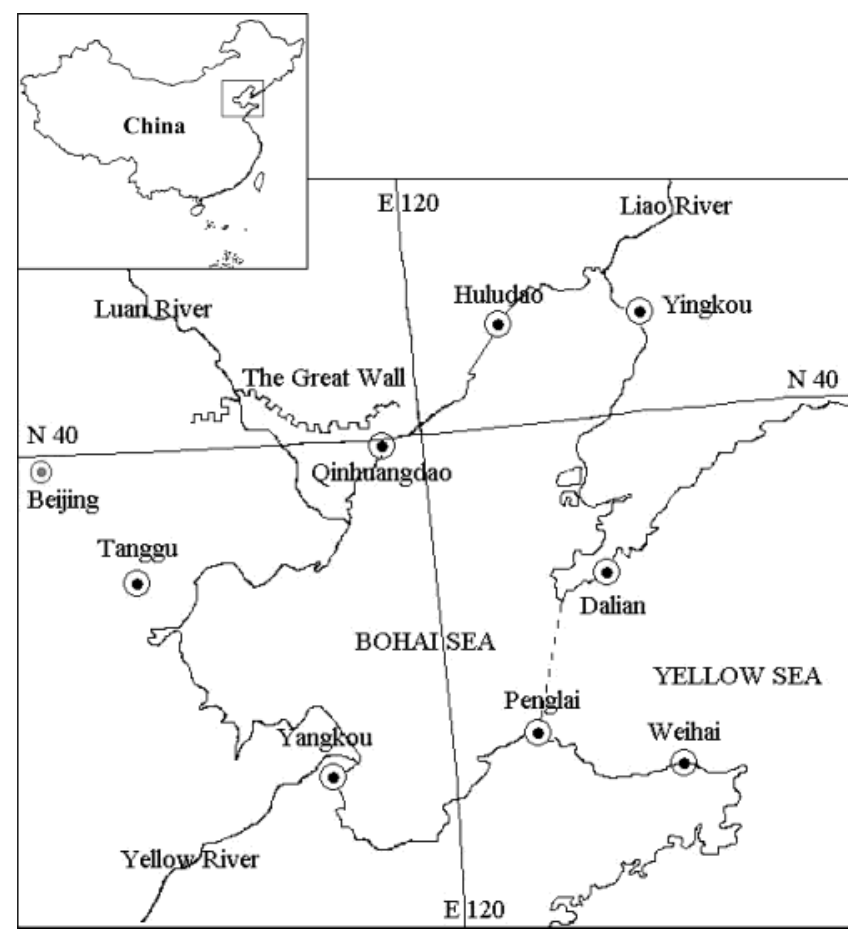

Figure 1. Sampling sites of mollusk samples along the Chinese Bohai Sea.

pump was substituted with a more competent peristaltic pump on the FIA-3100 analyzer (Beijing Wantuo Instrument Co., China), which was used to pump the $\mathrm{K}_{2} \mathrm{~S}_{2} \mathrm{O}_{8}$ oxidant solution and $\mathrm{KBH}_{4}$ reducing agent. The total mercury determination was made with reference to the previous method (5).

Reagents and Standards. All chemicals were of guaranteed reagent grade except where specified, and Milli-Q water was used throughout. Stock solutions of standard methylmercury chloride $\left(\mathrm{CH}_{3} \mathrm{HgCl}\right.$, Merck) and mercury chloride $\left(\mathrm{HgCl}_{2}\right.$, Merck $)\left(1 \mathrm{mg} \mathrm{ml}^{-1}\right.$ as $\left.\mathrm{Hg}\right)$ were prepared by dissolving appropriate amounts in methanol and $5 \% \mathrm{v} / \mathrm{v} \mathrm{HNO}_{3}$, respectively. The mercury working solutions were obtained by dilution with methanol or $10 \% \mathrm{v} / \mathrm{v} \mathrm{HNO}_{3}$ and prepared daily before use. All solutions were stored at $4{ }^{\circ} \mathrm{C}$.

Aqueous solutions, $0.1 \mathrm{~mol} \mathrm{l}^{-1}$ tetrabutylammonium bromide (TBA), $1 \mathrm{~mol} 1^{-1} \mathrm{NaCl}, 25 \% \mathrm{~m} / \mathrm{v} \mathrm{KOH} / \mathrm{CH}_{3} \mathrm{OH}$, and $10 \mathrm{mmol} 1^{-1} \mathrm{Na}_{2} \mathrm{~S}_{2} \mathrm{O}_{3}$ solution, were prepared weekly and stored at $4{ }^{\circ} \mathrm{C}$. Daily prepared HPLC mobile phase was a mixture of appropriate TBA, $\mathrm{NaCl}$ solution, water, and $\mathrm{CH}_{3} \mathrm{OH}$ and filtered through a $0.45-\mu \mathrm{m}$ membrane filter before use.

$\mathrm{KBH}_{4}$ solutions $(0.01 \% \mathrm{~m} / \mathrm{v}$ and $0.2 \% \mathrm{~m} / \mathrm{v})$ were prepared daily by dissolving the required amount in $0.2 \% \mathrm{~m} / \mathrm{v} \mathrm{KOH}$. An oxidant solution of $1 \% \mathrm{~m} / \mathrm{v} \mathrm{K}_{2} \mathrm{~S}_{2} \mathrm{O}_{8}$ solution was prepared in $10 \% \mathrm{v} / \mathrm{v} \mathrm{HCl}$.

Sampling. Figure 1 shows the eight sampling sites of mollusks. These sites spread out around the Bohai Sea. Mollusks with different dimensions were divided into several size groups, and the dimensions and numbers of mollusks were listed in Table $\mathbf{1}$ according to sampling sites and species. The soft tissues of mollusks were excised with stainless steel scalpel blades, thoroughly rinsed with Milli-Q water to remove extraneous impurities, and homogenized using a blender. The homogenized samples were kept at $-18^{\circ} \mathrm{C}$ until analysis.

Different species of mollusks were identified according to the catalog of marine mollusks in reference books (6).

Procedures. For methylmercury analysis, samples were prepared according to the published method with slight modification (7). Briefly, $2 \mathrm{~mL}$ of $25 \% \mathrm{~m} / \mathrm{v} \mathrm{KOH} / \mathrm{CH}_{3} \mathrm{OH}$ was added to $1.0-2.0$-g homogenized wet samples in a $50-\mathrm{mL}$ centrifuge tube and shaken mechanically overnight. Then $6 \mathrm{~mL}$ of $\mathrm{CH}_{2} \mathrm{Cl}_{2}$ was added, and $1.5 \mathrm{~mL}$ of concentrated $\mathrm{HCl}$ was dropped in sequence, followed by shaking for 10 minutes to extract organic mercury into the $\mathrm{CH}_{2} \mathrm{Cl}_{2}$ phase. After centrifuging at $2000 \mathrm{rpm}$ for $10 \mathrm{~min}$, the $4 \mathrm{~mL}$ of $\mathrm{CH}_{2} \mathrm{Cl}_{2}$ phase was transferred into a $10-\mathrm{mL}$ glass tube and extracted with $1 \mathrm{~mL}$ of sodium thiosulfate. Shaking for $45 \mathrm{~min}$ was needed to hasten the extraction speed. Another centrifugation at $5000 \mathrm{rpm}, 4^{\circ} \mathrm{C}$ for $15 \mathrm{~min}$, was necessary to separate the fat layer. The water phase was injected directly into the HPLCAFS system.

For total mercury analysis, approximately $1 \mathrm{~g}$ (wet weight) of soft tissues were directly weighed into a PTFE digestion container. Concentrated nitric acid $(3 \mathrm{~mL})$ was added, and the containers were sealed and left to predigest overnight on an electrothermal hotplate at $40{ }^{\circ} \mathrm{C}$. After cooling, $2 \mathrm{~mL}$ of hydrogen peroxide was added into the containers, which were placed in stainless steel bombs, sealed with a screw closure to avoid any acid leakage, and placed in an oven. The oven temperature was first raised to $50{ }^{\circ} \mathrm{C}$ and kept for $1 \mathrm{~h}$, then increased to $160^{\circ} \mathrm{C}$ for another $4 \mathrm{~h}$. After cooling to room temperature, the solutions were completely transferred into a 50-mL PET bottle and diluted with Milli-Q water, then determined by AFS method. Reagent blanks were processed simultaneously.

\section{RESULTS AND DISCUSION}

Evaluation of the Methods. Certified reference material DORM-2 (Dogfish muscle) was analyzed to validate the two analytical methods. As shown in Table 2, the experimental results were in good agreement with the certified values.

Mercury Contents in Mollusks. Contents of methylmercury and total mercury and the ratio of methylmercury to total mercury in gastropods and bivalves at each sampling site are shown in Tables 3 and 4, respectively. Mercury was detected in all samples. Methylmercury levels ranged from 4.8 to 168.4 $\mathrm{ng} \mathrm{Hg} \mathrm{g}^{-1}$, while total mercury contents ranged from 6.7 to $453.0 \mathrm{ng} \mathrm{Hg} \mathrm{g}^{-1}$. It was found that mollusks collected from different places contained different levels of methylmercury and total mercury. Roughly, samples collected from HuLuDao contained the highest level, those from PengLai took the second place, and those from the other six sampling sites contained the lowest level. On the basis of the analysis of Tables 3 and 4, gastropods showed higher bioaccumulation capacity for mercury than bivalves.

Methylmercury in Mollusks. Methylmercury contents in bivalves varied from 4.8 to $47.5 \mathrm{ng} \mathrm{Hg} \mathrm{g}^{-1}$. The methylmercury and total mercury contents in bivalves at the eight sampling sites are given in Table 4. Methylmercury levels in bivalves from HuLuDao and PengLai were similar and higher than those from the other sites. As for gastropods, the methylmercury contents were 4.9-168.4 $\mathrm{ng} \mathrm{Hg} \mathrm{g}^{-1}$, which was similar to those in bivalves. From Table 3, it was obvious that gastropods from HuLuDao contained the highest levels of methylmercury, while methylmercury contents in those from other sites differed slightly. For all the three-size group of Rapana venosa from HuLuDao, the methylmercury levels ranged from 40.9 to 168.4 $\mathrm{ng} \mathrm{g}^{-1}$, and the large Rapana venosa contained methylmercury content exceeding the maximum permissible levels (MPLs) of $0.16 \mathrm{mg} \mathrm{kg}^{-1}$ wet weight, which was established by the Ministry of Health of the State of Minnesota (USA). Comparing with the similar dimensions of Rapana venosa in other sampling sites, those from HuLuDao were the most seriously polluted.

Total Mercury in Mollusks. Total mercury contents in bivalves varied from 6.7 to $194.2 \mathrm{ng} \mathrm{Hg} \mathrm{g}^{-1}$. According to Table 4, the total mercury contamination levels of bivalves from HuLuDao and PengLai were similar and higher than those from the other sites. Considering the maximum permissible levels (MPLs) of total mercury in fish and shellfish set up by China $\left(0.3 \mathrm{mg} \mathrm{kg}^{-1}\right)$, Europe (EEC decision 93/351, $0.5 \mathrm{mg} \mathrm{kg}^{-1}$ ), and WHO (0.5 $\mathrm{mg} \mathrm{kg}^{-1}$ ), all the bivalves could be eaten with no worry about the hazards to human health. Mytilus edulis had medium mercury contents, but the one from PengLai showed extraor- 
Table 1. Dimension and Number for Three Size Classes (S-small, M-middle, L-large) of the Mollusks in Eight Sampling Sites: Bivalves (length) and Gastropods (height)

\begin{tabular}{|c|c|c|c|}
\hline species & denomination & dimension (mm) & no. \\
\hline \multicolumn{4}{|l|}{ WeiHai } \\
\hline \multirow{2}{*}{ gastropods } & Rapana venosa, Valenciennes, 1846 & $95 \pm 1$ & 2 \\
\hline & Neverita didyma, Röding, 1798 & $41 \pm 3$ & 5 \\
\hline \multirow[t]{8}{*}{ bivalves } & Ruditapes philippinarum, Adams \& Reeve, 1850 & $34 \pm 2$ & 22 \\
\hline & Meretix meretrix, Linnaeus, 1758 & $54 \pm 2$ & 7 \\
\hline & Sinonovacula constricta, Lamarck, 1818 & $57 \pm 2$ & 14 \\
\hline & Amusium, Röding. 1798 & $80 \pm 6$ & 6 \\
\hline & Chlamys (Azumapecten) farreri, Jones \& Preston, 1904 & $51 \pm 4$ & 8 \\
\hline & Crassostrea talienwhanensis, Crosse, 1862 & $64 \pm 11$ & 4 \\
\hline & Mytilus edulis, Linnaeus, 1758 & $58 \pm 4$ & 12 \\
\hline & Mya arenaria, Linnaeus, 1758 & $66 \pm 5$ & 3 \\
\hline \multicolumn{4}{|l|}{ PengLai } \\
\hline \multirow[t]{5}{*}{ gastropods } & Neptunea arthritica cumingii, Crosse, 1862 & $87 \pm 3$ & 4 \\
\hline & Rapana venosa, Valenciennes, $1846(\mathrm{~L})$ & $95 \pm 0$ & 2 \\
\hline & Rapana venosa, Valenciennes, $1846(\mathrm{~S})$ & $57 \pm 2$ & 7 \\
\hline & Neverita didyma. Röding. 1798(L) & $46 \pm 4$ & 2 \\
\hline & Neverita didyma, Röding, 1798(S) & $21 \pm 1$ & 12 \\
\hline \multirow{9}{*}{ bivalves } & Mactra (Mactra) veneriformis, Reeve, 1854 & $35 \pm 1$ & 14 \\
\hline & Ruditapes philippinarum. Adams \& Reeve, 1850 & $33+2$ & 20 \\
\hline & Meretix meretrix, Linnaeus, 1758 & $77 \pm 1$ & 7 \\
\hline & Scapharca subcrenata, Lischke, 1869 & $33 \pm 2$ & 15 \\
\hline & Amusium, Röding, 1798 & $106 \pm 2$ & 2 \\
\hline & Chlamys (Azumapecten) farreri, Jones \& Preston, 1904 & $51 \pm 4$ & 13 \\
\hline & Crassostrea talienwhanensis, Crosse, 1862 & $49 \pm 4$ & 6 \\
\hline & Mytilus edulis, Linnaeus, 1758 & $59 \pm 5$ & 8 \\
\hline & Mya arenaria, Linnaeus, 1758 & $74 \pm 1$ & 3 \\
\hline \multicolumn{4}{|l|}{ YangKou } \\
\hline \multirow[t]{3}{*}{ gastropods } & Rapana venosa, Valenciennes, 1846 & $96 \pm 6$ & 2 \\
\hline & Neverita didyma, Röding, 1798(L) & $31 \pm 0$ & 11 \\
\hline & Neverita didyma, Röding, 1798(S) & $18 \pm 1$ & 23 \\
\hline \multirow[t]{6}{*}{ bivalves } & Mactra (Mactra) veneriformis, Reeve, 1854 & $32 \pm 3$ & 12 \\
\hline & Ruditapes philippinarum, Adams \& Reeve, 1850 & $43 \pm 4$ & 9 \\
\hline & Meretix meretrix, Linnaeus, 1758 & $50 \pm 3$ & 10 \\
\hline & Sinonovacula constricta, Lamarck, 1818 & $54 \pm 2$ & 11 \\
\hline & Scapharca subcrenata, Lischke, 1869(L) & $54 \pm 6$ & 3 \\
\hline & Scapharca subcrenata, Lischke, 1869(S) & $35 \pm 2$ & 6 \\
\hline \multicolumn{4}{|l|}{ TangGu } \\
\hline gastropods & Rapana venosa, Valenciennes, 1846(L) & 119 & 1 \\
\hline & Rapana venosa, Valenciennes, $1846(\mathrm{M})$ & $68 \pm 4$ & 3 \\
\hline & Rapana venosa, Valenciennes, $1846\left(S^{\prime}\right)$ & $40 \pm 4$ & 11 \\
\hline & Neverita didyma, Röding, $1798(\mathrm{~L})$ & $26 \pm 2$ & 12 \\
\hline & Neverita didyma, Röding, 1798(S) & $16 \pm 2$ & 43 \\
\hline bivalves & Mactra (Mactra) veneriformis, Reeve, 1854 & $30 \pm 2$ & 27 \\
\hline & Meretix meretrix, Linnaeus, 1758 & $42 \pm 3$ & 10 \\
\hline & Sinonovacula constricta, Lamarck, 1818 & $55 \pm 2$ & 17 \\
\hline & Scapharca subcrenata, Lischke, 1869 & $49 \pm 2$ & 7 \\
\hline & Amusium, Röding, 1798 & $108 \pm 1$ & 2 \\
\hline & Crassostrea talienwhanensis, Crosse, 1862 & $63 \pm 4$ & 5 \\
\hline & Mya arenaria, Linnaeus, $1758(\mathrm{~L})$ & $100 \pm 5$ & 3 \\
\hline & Mya arenaria, Linnaeus, $1758(S)$ & $68 \pm 2$ & 4 \\
\hline QinHuangDao & & & \\
\hline gastropods & Rapana venosa, Valenciennes, $1846(\mathrm{~L})$ & $81 \pm 2$ & 3 \\
\hline & Rapana venosa, Valenciennes, $1846(\mathrm{~S})$ & $54 \pm 3$ & 5 \\
\hline & Neverita didyma, Röding, 1798 & $45 \pm 1$ & 4 \\
\hline bivalves & Mactra (Mactra) veneriformis, Reeve, 1854 & $32 \pm 2$ & 18 \\
\hline & Ruditapes philippinarum, Adams \& Reeve, 1850 & $28 \pm 2$ & 63 \\
\hline & Meretix meretrix, Linnaeus, 1758 & $55 \pm 3$ & 7 \\
\hline & Sinonovacula constricta, Lamarck, 1818 & $57 \pm 2$ & 14 \\
\hline & Scapharca subcrenata, Lischke, 1869 & $49 \pm 1$ & 4 \\
\hline & Amusium, Röding, 1798 & $40 \pm 2$ & 18 \\
\hline & Chlamys (Azumapecten) farreri, Jones \& Preston, 1904 & $68 \pm 3$ & 7 \\
\hline & Crassostrea talienwhanensis, Crosse, 1862 & $49 \pm 2$ & 7 \\
\hline & Mytilus edulis, Linnaeus, 1758 & $68 \pm 5$ & 16 \\
\hline HuLuDao & & & \\
\hline gastropods & Rapana venosa, Valenciennes, $1846(\mathrm{~L})$ & $84 \pm 5$ & 2 \\
\hline & Rapana venosa, Valenciennes, 1846(M) & $66 \pm 2$ & 4 \\
\hline & Rapana venosa, Valenciennes, $1846\left(S^{\prime}\right)$ & $44 \pm 2$ & 11 \\
\hline & Neverita didyma, Röding, 1798(L) & $56 \pm 1$ & 3 \\
\hline & Neverita didyma, Röding, 1798(M) & $38 \pm 4$ & 4 \\
\hline & Neverita didyma, Röding, 1798(S) & $24 \pm 2$ & 8 \\
\hline bivalves & Mactra (Mactra) veneriformis, Reeve, 1854 & $34 \pm 2$ & 18 \\
\hline & Ruditapes philippinarum, Adams \& Reeve, 1850 & $32 \pm 2$ & 42 \\
\hline & Meretix meretrix, Linnaeus, 1758 & $59 \pm 2$ & 5 \\
\hline & Scapharca subcrenata, Lischke, 1869 & $40 \pm 5$ & 5 \\
\hline
\end{tabular}


Table 1. Cont.

\begin{tabular}{|c|c|c|c|}
\hline species & denomination & dimension (mm) & no. \\
\hline $\begin{array}{l}\text { YingKou } \\
\text { gastropods } \\
\text { bivalves }\end{array}$ & $\begin{array}{l}\text { Rapana venosa, Valenciennes, } 1846 \\
\text { Mactra (Mactra) veneriformis, Reeve, } 1854 \\
\text { Ruditapes philippinarum, Adams \& Reeve, } 1850 \\
\text { Meretix meretrix, Linnaeus, } 1758 \\
\text { Sinonovacula constricta, Lamarck, } 1818 \\
\text { Amusium, Röding, } 1798 \\
\text { Mytilus edulis, Linnaeus, } 1758\end{array}$ & $\begin{array}{l}69 \pm 5 \\
30 \pm 2 \\
37 \pm 2 \\
52 \pm 3 \\
48 \pm 2 \\
68 \pm 3 \\
71 \pm 7\end{array}$ & $\begin{array}{r}4 \\
22 \\
18 \\
7 \\
33 \\
5 \\
6\end{array}$ \\
\hline DaLian & & & \\
\hline gastropods & $\begin{array}{l}\text { Neptunea arthritica cumingii, Crosse, } 1862(\mathrm{~L}) \\
\text { Neptunea arthritica cumingii, Crosse, } 1862(\mathrm{M}) \\
\text { Neptunea arthritica cumingii, Crosse, 1862(S) } \\
\text { Neverita didyma, Röding, } 1798\end{array}$ & $\begin{array}{r}122 \pm 1 \\
70 \pm 0 \\
42 \pm 3 \\
38 \pm 2\end{array}$ & $\begin{array}{r}2 \\
5 \\
14 \\
10\end{array}$ \\
\hline bivalves & $\begin{array}{l}\text { Mactra (Mactra) veneriformis, Reeve, } 1854 \\
\text { Ruditapes philippinarum, Adams \& Reeve, } 1850 \\
\text { Meretix meretrix, Linnaeus, } 1758 \\
\text { Sinonovacula constricta, Lamarck, } 1818 \\
\text { Scapharca subcrenata, Lischke, } 1869 \\
\text { Amusium, Röding, } 1798 \\
\text { Chlamys (Azumapecten) farreri, Jones \& Preston, } 1904 \\
\text { Crassostrea talienwhanensis, Crosse, } 1862 \\
\text { Mytilus edulis, Linnaeus, } 1758\end{array}$ & $\begin{array}{l}32 \pm 1 \\
30 \pm 3 \\
50 \pm 3 \\
52 \pm 2 \\
34 \pm 2 \\
72 \pm 5 \\
50 \pm 4 \\
59 \pm 7 \\
61 \pm 5\end{array}$ & $\begin{array}{r}12 \\
46 \\
9 \\
29 \\
16 \\
5 \\
11 \\
6 \\
21\end{array}$ \\
\hline
\end{tabular}

Table 2. Results of Methylmercury (MeHg) and Total Mercury (HgT) Contents in DORM-2 (Dogfish Muscle)

\begin{tabular}{|c|c|c|}
\hline sample & $\begin{array}{c}\text { determination values } \\
\left(\text { mean }^{a} \pm \text { S. D. } .^{b}, \text { ng g }^{-1}\right)\end{array}$ & $\begin{array}{c}\text { certified values } \\
\left(\text { mean }^{a} \pm \text { S. D. } .^{b}, \mathrm{ng} \mathrm{g}^{-1}\right)\end{array}$ \\
\hline $\begin{array}{l}\text { MeHg concentration } \\
\left(\text { mean }^{a} \pm \text { S. D. }{ }^{b} \text { ng g }^{-1}\right)^{c}\end{array}$ & $4237 \pm 143$ & $4470 \pm 320$ \\
\hline $\begin{array}{l}\text { HgT concentration } \\
\left(\text { mean }^{a} \pm \text { S. D. }{ }^{b}, \mathrm{ng} \mathrm{g}^{-1}\right)^{d}\end{array}$ & $4560 \pm 460$ & $4640 \pm 260$ \\
\hline
\end{tabular}
AFS.

${ }^{a} N=3 .{ }^{b}$ Standard deviation. ${ }^{c}$ Determined by HPLC-AFS. ${ }^{d}$ Determined by

dinarily high mercury content. It was supposed that this sample was collected from an extremely mercury-polluted place, and this was also supported by the more severe contamination statues in bivalves from PengLai. As for gastropods, the total mercury contents ranged from 11.3 to $453.0 \mathrm{ng} \mathrm{Hg} \mathrm{g}^{-1}$. Table 3 indicates that mercury contamination level in gastropods from HuLuDao was at least twice that of those from PengLai and other sites. Among the 88 samples, only large Rapana venosa in HuLuDao contained high levels of total mercury that went beyond the MPLs set up by China. However, much attention should be paid when consuming a large amount of this species of seafood in a short period. When the total mercury contents in bivalves and gastropods were compared, it was easy to find the great difference between them, which just illuminated the distinct mercury bioaccumulation capacity of them. Maybe this is because the bivalves are grass-eating mollusks, while the three collected gastropod species are all predatory flesh-eating mollusks, the main food of which are bivalves. So from the view of trophic

Table 3. Methylmercury (MeHg), Total Mercury $(\mathrm{HgT})$, and the MeHg/HgT Ratio in Gastropods Collected from Eight Coastal Cities in China (Wet Weight, $\mathrm{ng} \mathrm{g}^{-1}$ )

\begin{tabular}{|c|c|c|c|c|}
\hline sites & denomination & $\mathrm{MeHg}$ & $\mathrm{HgT}$ & $\mathrm{MeHg} / \mathrm{Hg} \mathrm{T}(\%)$ \\
\hline \multirow[t]{2}{*}{ WeiHai } & Rapana venosa, Valenciennes, 1846 & 18.6 & 42.4 & 44 \\
\hline & Neverita didyma, Röding, 1798 & 14.1 & 33.0 & 43 \\
\hline \multirow{5}{*}{ PengLai } & Neptunea arthritica cumingii, Crosse, 1862 & 27.6 & 94.1 & 29 \\
\hline & Rapana venosa. Valenciennes, $1846(\mathrm{~L})$ & 19.1 & 97.9 & 19 \\
\hline & Rapana venosa, Valenciennes, $1846(\mathrm{~S})$ & 18.1 & 63.0 & 29 \\
\hline & Neverita didyma, Röding, $1798(\mathrm{~L})$ & 44.2 & 138.7 & 32 \\
\hline & Neverita didyma, Röding, 1798(S) & 38.5 & 62.2 & 62 \\
\hline \multirow[t]{3}{*}{ YangKou } & Rapana venosa. Valenciennes, 1846 & 18.8 & 59.1 & 32 \\
\hline & Neverita didyma. Röding. 1798(L) & 23.2 & 96.1 & 24 \\
\hline & Neverita didyma, Röding, 1798(S) & 16.1 & 32.0 & 50 \\
\hline \multirow[t]{5}{*}{ TangGu } & Rapana venosa, Valenciennes, $1846(\mathrm{~L})$ & 14.9 & 37.8 & 40 \\
\hline & Rapana venosa, Valenciennes, $1846(\mathrm{M})$ & 10.5 & 24.0 & 44 \\
\hline & Rapana venosa, Valenciennes, $1846(\mathrm{~S})$ & 4.9 & 11.3 & 44 \\
\hline & Neverita didyma. Röding, $1798(\mathrm{~L})$ & 10.4 & 26.6 & 39 \\
\hline & Neverita didyma, Röding. 1798(S) & 13.0 & 42.7 & 30 \\
\hline \multirow{3}{*}{ QinHuangDao } & Rapana venosa, Valenciennes, $1846(\mathrm{~L})$ & 13.8 & 41.7 & 33 \\
\hline & Rapana venosa, Valenciennes, $1846(\mathrm{~S})$ & 16.1 & 53.4 & 30 \\
\hline & Neverita didyma, Röding, 1798 & 17.8 & 44.0 & 40 \\
\hline \multirow[t]{6}{*}{ HuLuDao } & Rapana venosa, Valenciennes, $1846(\mathrm{~L})$ & 168.4 & 453.0 & 37 \\
\hline & Rapana venosa, Valenciennes, $1846(\mathrm{M})$ & 55.9 & 276.7 & 20 \\
\hline & Rapana venosa. Valenciennes, 1846 (S) & 40.9 & 199.2 & 21 \\
\hline & Neverita didyma, Röding, $1798(\mathrm{~L})$ & 49.9 & 128.0 & 39 \\
\hline & Neverita didyma, Röding, 1798(M) & 34.3 & 77.2 & 44 \\
\hline & Neverita didyma, Röding, 1798(S) & 29.7 & 53.1 & 56 \\
\hline YingKou & Rapana venosa, Valenciennes, 1846 & 24.4 & 60.5 & 40 \\
\hline \multirow[t]{4}{*}{ DaLian } & Neptunea arthritica cumingii, Crosse, $1862(\mathrm{~L})$ & 25.2 & 109.5 & 23 \\
\hline & Neptunea arthritica cumingii. Crosse, $1862(\mathrm{M})$ & 17.5 & 67.2 & 26 \\
\hline & Neptunea arthritica cumingii. Crosse, 1862 (S) & 20.2 & 83.3 & 24 \\
\hline & Neverita didyma, Röding, 1798 & 14.2 & 39.3 & 36 \\
\hline
\end{tabular}


Table 4. Methylmercury (MeHg), Total Mercury (HgT), and the MeHg/HgT Ratio in Bivalves Collected from Eight Coastal Cities in China (Wet Weight, $n g g^{-1}$ )

\begin{tabular}{|c|c|c|c|c|}
\hline sites & denomination & $\mathrm{MeHg}$ & $\mathrm{HgT}$ & $\mathrm{MeHg} / \mathrm{HgT}(\%)$ \\
\hline \multirow[t]{8}{*}{ WeiHai } & Ruditapes philippinarum, Adams \& Reeve, 1850 & 8.4 & 14.8 & 57 \\
\hline & Meretix meretrix, Linnaeus, 1758 & 8.0 & 9.1 & 87 \\
\hline & Sinonovacula constricta, Lamarck, 1818 & 7.3 & 10.4 & 70 \\
\hline & Amusium, Röding, 1798 & 13.9 & 20.2 & 69 \\
\hline & Chlamys (Azumapecten) farreri, Jones \& Preston, 1904 & 11.6 & 17.1 & 68 \\
\hline & Crassostrea talienwhanensis, Crosse, 1862 & 8.2 & 15.7 & 52 \\
\hline & Mytilus edulis, Linnaeus, 1758 & 9.0 & 11.2 & 81 \\
\hline & Mya arenaria, Linnaeus, 1758 & 13.8 & 18.1 & 76 \\
\hline \multirow[t]{9}{*}{ PengLai } & Mactra (Mactra) veneriformis, Reeve, 1854 & 15.6 & 19.7 & 80 \\
\hline & Ruditapes philippinarum, Adams \& Reeve, 1850 & 21.3 & 26.9 & 79 \\
\hline & Meretix meretrix, Linnaeus, 1758 & 47.5 & 92.1 & 52 \\
\hline & Scapharca subcrenata, Lischke, 1869 & 26.5 & 44.1 & 60 \\
\hline & Amusium, Röding, 1798 & 16.6 & 23.3 & 71 \\
\hline & Chlamys (Azumapecten) farreri, Jones \& Preston, 1904 & 22.8 & 34.0 & 67 \\
\hline & Crassostrea talienwhanensis, Crosse, 1862 & 37.0 & 64.4 & 58 \\
\hline & Mytilus edulis, Linnaeus, 1758 & 40.6 & 194.2 & 21 \\
\hline & Mya arenaria, Linnaeus, 1758 & 16.6 & 24.4 & 68 \\
\hline \multirow{6}{*}{ YangKou } & Mactra (Mactra) veneriformis, Reeve, 1854 & 12.3 & 20.7 & 59 \\
\hline & Ruditapes philippinarum, Adams \& Reeve, 1850 & 12.5 & 32.5 & 38 \\
\hline & Meretix meretrix, Linnaeus, 1758 & 10.4 & 16.1 & 65 \\
\hline & Sinonovacula constricta, Lamarck, 1818 & 8.4 & 10.7 & 79 \\
\hline & Scapharca subcrenata, Lischke, 1869(L) & 18.0 & 45.4 & 40 \\
\hline & Scapharca subcrenata, Lischke, 1869(S) & 27.7 & 68.4 & 41 \\
\hline \multirow{8}{*}{ TangGu } & Mactra (Mactra) veneriformis, Reeve, 1854 & 5.6 & 7.8 & 72 \\
\hline & Meretix meretrix, Linnaeus, 1758 & 4.8 & 6.7 & 72 \\
\hline & Sinonovacula constricta, Lamarck, 1818 & 8.7 & 9.8 & 89 \\
\hline & Scapharca subcrenata, Lischke, 1869 & 10.4 & 16.4 & 63 \\
\hline & Amusium, Röding, 1798 & 10.8 & 22.4 & 48 \\
\hline & Crassostrea talienwhanensis, Crosse, 1862 & 11.7 & 15.6 & 75 \\
\hline & Mya arenaria, Linnaeus, $1758(\mathrm{~L})$ & 11.6 & 16.1 & 72 \\
\hline & Mya arenaria, Linnaeus, $1758(S)$ & 21.8 & 51.3 & 42 \\
\hline \multirow[t]{9}{*}{ QinHuangDao } & Mactra (Mactra) veneriformis, Reeve, 1854 & 13.0 & 16.9 & 77 \\
\hline & Ruditapes philippinarum, Adams \& Reeve, 1850 & 13.9 & 18.6 & 75 \\
\hline & Meretix meretrix, Linnaeus, 1758 & 7.5 & 12.6 & 59 \\
\hline & Sinonovacula constricta, Lamarck, 1818 & 14.4 & 19.6 & 74 \\
\hline & Scapharca subcrenata, Lischke, 1869 & 11.2 & 13.9 & 81 \\
\hline & Amusium, Röding, 1798 & 9.7 & 13.4 & 73 \\
\hline & Chlamys (Azumapecten) farreri, Jones \& Preston, 1904 & 14.5 & 19.7 & 74 \\
\hline & Crassostrea talienwhanensis, Crosse, 1862 & 14.8 & 18.1 & 82 \\
\hline & Mytilus edulis, Linnaeus, 1758 & 8.9 & 11.0 & 80 \\
\hline \multirow[t]{4}{*}{ HuLuDao } & Mactra (Mactra) veneriformis, Reeve, 1854 & 25.2 & 77.8 & 32 \\
\hline & Ruditapes philippinarum, Adams \& Reeve, 1850 & 24.4 & 38.2 & 64 \\
\hline & Meretix meretrix, Linnaeus, 1758 & 15.8 & 19.9 & 80 \\
\hline & Scapharca subcrenata, Lischke, 1869 & 38.4 & 99.3 & 39 \\
\hline \multirow[t]{6}{*}{ YingKou } & Mactra (Mactra) veneriformis, Reeve, 1854 & 18.7 & 43.2 & 43 \\
\hline & Ruditapes philippinarum, Adams \& Reeve, 1850 & 15.7 & 27.8 & 56 \\
\hline & Meretix meretrix, Linnaeus, 1758 & 8.6 & 15.0 & 57 \\
\hline & Sinonovacula constricta, Lamarck, 1818 & 23.6 & 28.4 & 83 \\
\hline & Amusium, Röding, 1798 & 18.8 & 27.3 & 69 \\
\hline & Mytilus edulis, Linnaeus, 1758 & 19.6 & 29.4 & 67 \\
\hline \multirow{9}{*}{ DaLian } & Mactra (Mactra) veneriformis, Reeve, 1854 & 12.6 & 30.4 & 41 \\
\hline & Ruditapes philippinarum, Adams \& Reeve, 1850 & 6.4 & 11.1 & 58 \\
\hline & Meretix meretrix, Linnaeus, 1758 & 16.8 & 25.1 & 67 \\
\hline & Sinonovacula constricta, Lamarck, 1818 & 15.6 & 24.7 & 63 \\
\hline & Scapharca subcrenata, Lischke, 1869 & 14.4 & 26.2 & 55 \\
\hline & Amusium, Röding, 1798 & 15.3 & 28.2 & 54 \\
\hline & Chlamys (Azumapecten) farreri, Jones \& Preston, 1904 & 17.1 & 30.5 & 56 \\
\hline & Crassostrea talienwhanensis, Crosse, 1862 & 13.0 & 24.1 & 54 \\
\hline & Mytilus edulis, Linnaeus, 1758 & 17.1 & 30.9 & 55 \\
\hline
\end{tabular}

levels, the gastropods are superior to bivalves and can bioaccumulate mercury effectively.

Combining with the above-mentioned comparison of methylmercury and total mercury content in mollusks from the eight sampling sites, we could easily find the serious mercury contamination levels in mollusks from HuLuDao. In HuLuDao city, the biggest zinc plant in Asia, built in the 1930s', continually pours wastewater and waste residue containing heavy metals into the Bohai Sea. It causes the excess $\mathrm{Hg}, \mathrm{Zn}, \mathrm{Cu}, \mathrm{Cd}$, and $\mathrm{Pb}$ deposition in the sediments and halobios. Further- more, the wastewater from the oil refining plant, chemical plant, and petrochemical industry in JinXi city, on the west of HuLuDao city, contribute to the mercury pollution in the mollusks. The wastewater flows through the WuLi River and finally pours into the Bohai Sea. It was reported that, from the entrance of the WuLi River to the Bohai Sea, the deposited mercury had reached 90 tons. Many halobios have been killed out, and the living halobios cannot be eaten any more. As for PengLai, the mercury pollution in mollusks attribute to the paper mill and the gold mines in the adjacent area. Wastewater from 
the paper mill is dumped into the Bohai Sea with no disposal. Also, the private industrial owners extract gold by the goldmercury amalgam method, which causes the local mercury pollution in rivers and soils, and all the mercury converges to the Bohai Sea at last.

Bioaccumulation of Mercury in Mollusks. Three species of gastropods and two species of bivalves were collected with different sizes. According to the different dimensions in each sampling site, the three gastropods were roughly divided into three-size classes (i.e., the L (large), M (middle), and S (small) classes). Two bivalve samples, Scapharca subcrenata and Mya arenaria, were divided into L (large) and S (small) size classes. The results listed in Table $\mathbf{3}$ showed that mercury bioaccumulation in different sizes of gastropods differed evidently. Mercury in Rapana venosa showed uplifted trends with the increasing of the shells' size. Rapana venosa from three sites presented the elevated $\mathrm{Hg}$ content, especially in the more severely contaminated samples collected from HuLuDao. However, Rapana venosa from QinHuangDao did not agree with this trend, probably because the growth dilution effect played an important role. Neverita didyma also showed similar variability to Rapana venosa. Three dimensions of Neptunea arthritica cumingii were only collected in DaLian and the accretion trend was not very clear. Other than Rapana venosa and Neverita didyma, Scapharca subcrenata and Mya arenaria did not show the uplifted trends. However, this conclusion was unilateral for lacking of sufficient data. The highest mercury level and the infinite bioaccumulation in the Rapana venosa soft tissue might indicate that it is a potential biomarker to monitor the mercury pollution in oceanic ecosystems.

The Ratio of Methylmercury to Total Mercury. The proportions of methylmercury to total mercury in bivalves ranged from 20.9 to $89.3 \%$, which was in accord with the previous reports. Claisse et al. reported a methylmercury/total mercury ratio from 21 to $74 \%$ in mussels and oysters along the French Coast (8). Mikac et al. reported a methylmercury/total mercury ratio of about $40 \%$ in mussels from the Krka Estuary (Croatia) (9) in 1996. While for gastropod species, the ratio of methylmercury to total mercury was in the range of 19.5 to $62.0 \%$. However, no literature has reported the ratio of methylmercury to total mercury in gastropods. This ratio in gastropods was lower than that in bivalves, according to our results. The different proportions of methylmercury to total mercury in bivalves and gastropods may correlate with their different absorption mechanism and metabolism. Gastropods accumulated more mercury than bivalves, and to protect themselves from hazards, there might be an automatic biotransformation process, in which methylmercury was converted into less toxic inorganic forms $(10-11)$.

The different ratios among different sampling sites might correspond to the local methylation conditions in seawaters and sediments, such as temperature, salinity, $\mathrm{pH}$, content of organic matters, numbers, and species of bacteria, which might lead to the different conversion percentage from inorganic mercury to methylmercury.

The contamination levels of methylmercury and total mercury in 13 species of mollusks and gastropods sampled from eight coastal sites along the Bohai Sea were investigated. Mercury were detected in all samples, and gastropods showed more capacity to bioaccumulate mercury than bivalves. The results also indicated that mercury contents in two gastropods presented uplifted trends with the increasing of the shells' dimensions. Due to the $\mathrm{Hg}$ drainage from the chemical industries in the adjacent area, mollusks collected from HuLuDao were obviously the most severely polluted samples. Comparing with the $\mathrm{Hg}$ contents in Rapana venosa in seven sampling sites, we found that Rapana venosa could not regulate $\mathrm{Hg}$ contents in its body and might be used as a potential biomarker to monitor $\mathrm{Hg}$ pollution in oceans.

\section{ACKNOWLEDGMENT}

We acknowledge Professor F. C. Adams for his kindly offering CRM DORM-2.

\section{LITERATURE CITED}

(1) Westöö, G. Determination of methylmercury compounds in foods. I. Methylmercury compounds in fish; identification and determination. Acta Chem. Scand. 1966, 20 (8), 2131-2137.

(2) Hintelmann, H.; Wilken, R. D. The analysis of organic mercury compounds using liquid chromatography with on-line atomic fluorescence spectrometric detection. Appl. Organomet. Chem. 1993, 7 (3), 173-180.

(3) Ramalhosa, E.; Río Segade, S.; Pereira, E.; Vale, C.; Duarte, A. Simple methodology for methylmercury and inorganic mercury determinations by high-performance liquid chromatography-cold vapour atomic fluorescence spectrometry. Anal. Chim. Acta 2001, 448, 135-143.

(4) Liang, L. N.; Jiang, G. B.; Liu, J. F.; Hu, J. T. Speciation analysis of mercury in seafood by using high-performance liquid chromatography on-line coupled with cold-vapor atomic fluorescence spectrometry via a postcolumn microwave digestion. Anal. Chim. Acta 2003, 477 (1), 131-137.

(5) Liang, L. N.; Jiang, G. B.; Hu, J. T. Determination of inorganic mercury and total organomercury in chemical wastewater by cold vapor generation-atomic fluorescence spectrometry. Chin. J. Anal. Chem. 2001, 29 (4), 403-405.

(6) Qi, Z. Y.; Ma, X. T.; Wang, Z. R.; et al. Mollusca of Huanghai and Bohai; Agriculture Publishing House: Beijing, China, 1989.

(7) Liang, L.; Horvat, M.; Cernichiari, E.; Gelein, B.; Balogh, S. Simple solvent extraction technique for elimination of matrix interferences in the determination of methylmercury in environmental and biological samples by ethylation-gas chromatographycold vapor atomic fluorescence spectrometry. Talanta 1996, 43 (11), 1883-1888.

(8) Claisse, D.; Cossa, D.; Bretaudeausanjuan, J.; Touchard, G.; Bombled, B. Methylmercury in mollusks along the French coast. Mar. Pollut. Bull. 2001, 42 (4), 329-332.

(9) Mikac, N.; Kwokal, Z.; Martincic, D.; Branica, M. Uptake of mercury species by transplanted mussels Mytilus galloprovincialis under estuarine conditions Krka River estuary. Sci. Total Environ. 1996, 184, 173-182.

(10) Nigro, M. Mercury and selenium localization in macrophages of the striped dolphin, Stenella coeruleoalba. J. Mar. Bio. Assoc. 1994, UK 74, 975-978.

(11) Nigro, M.; Leonzio, C. Intracellular storage of mercury and selenium in different marine vertebrates. Mar. Ecol. Prog. Ser. 1996, 135, 137-143.

Received for review February 27, 2003. Revised manuscript received September 25, 2003. Accepted September 30, 2003. This work was jointly supported by National Natural Science Foundation of China(20137010, 20205008) and Chinese Academy of Sciences (KZCX2-414).

JF034199L 